\title{
Effect of antibiotics on implant failure and postoperative infection
}

\author{
Abstracted from \\ Ata-Ali J, Ata-Ali F, Ata-Ali F.
}

Do antibiotics decrease implant failure and postoperative infections? A systematic review and meta-analysis. Int J Oral Maxillifac Surg 2014; 43: 68-74.

Address for correspondence: Javier Ata-Ali, Valencia University Medical and Dental School Public Dental Health Service. Arnau de Vilanova Hospital San Clemente Street 12 46015-Valencia, Spain. E-mail: javiataali@hotmail.com

Question: Among patients receiving dental implants, does the use of antibiotics, when compared with a control group, reduce the frequency of implant failure and postoperative infection?

Data sources Medline and hand search of the British Journal of Oral and Maxillofacial Surgery, Clinical Implant Dentistry and Related Research, Clinical Oral Investigations, Clinical Oral Implants Research, European Journal of Oral Implantology, Implant Dentistry, International Journal of Oral and Maxillofacial Implants, International Journal of Oral and Maxillofacial Surgery, Journal of Clinical Periodontology, Journal of Oral Implantology, Journal of Oral and Maxillofacial Surgery, Journal of Periodontology Medicina Oral, Patología Oral y Cirugía Bucal, and Oral Surgery, Oral Medicine, Oral Pathology, Oral Radiology and Endodontology.

Study selection Search of the literature was made to identify randomised controlled trials (RCTs) on the efficacy of antibiotics compared with a control group (not receiving antibiotics or receiving placebo.

Data extraction and synthesis All articles selected from the electronic and manual searches were independently assessed by the first and second authors of this study, in accordance with the established inclusion criteria. Two reviewers independently and in duplicate evaluated the quality of the included RCTs as part of the data extraction process.

Results Four RCTs were included in the final review. These four RCTs grouped a total of 2063 implants and a total of 1002 patients. Conclusions Antibiotic use significantly lowered the implant failure rate $(P=0.003)$, with an odds ratio of 0.331 , implying that antibiotic treatment reduced the odds of failure by $66.9 \%$. The number needed to treat (NNT) to prevent one patient from having an implant failure was 48 (95\% confidence interval 31-109). In contrast, antibiotic use did not significantly reduce the incidence of postoperative infection $(P=0.754)$. Based on the results of this meta-analysis, and pending further research in the field, it can be concluded that there is evidence in favour of systematic antibiotic use in patients receiving dental implants, since such treatment significantly reduces implant failure. In contrast, antibiotic use does not exert a significant preventive effect against postoperative infection.

\section{Commentary}

Dental implants are the treatment of choice for most missing teeth scenarios because of the reported high success rate and patient preferences. As with all surgical procedures, implant placement may be associated with the potential of complications arising during or after surgery. So far, mainly antibiotics are being used to reduce these complications.

This systematic review aims to investigate the effect of antibiotics in reducing the implant failure and postoperative infection rate when compared with a control group.

The authors included randomised clinical trials (RCTs) published to December of 2012 from a search of a single database and hand searching with no language restrictions. The authors also followed the Quality of Reporting of Meta-analysis (QUOROM) statement and two independent reviewers evaluated the quality of the included RCTs.

The primary outcome: implant failure was evaluated in four RCTs. Also four studies were included in the evaluation of the secondary outcome: postoperative infection rate. Risk of bias was determined from the included studies. Results were combined despite each study defining failure and infection differently and the antibiotic regimen not being the same.

The review concluded that the use of antibiotics significantly reduces implant failure but shows no superiority on postoperative infections. This is in agreement with the findings of the high quality systematic review from Cochrane on the same subject. ${ }^{1}$ Risk benefits of antibiotics use may need to be reconsidered altogether in light of the recent WHO report on bacterial resistance. $^{2}$

Arwa S Bafail, Ahmed M Alamri and Silvia Spivakovsky NYU College of Dentistry, New York, USA

1. Esposito M, Grusovin MG, Worthington HV. Interventions for replacing missing teeth: antibiotics at dental implant placement to prevent complications. Cochrane Database Syst Rev 2013; 7: CD004152. doi: 10.1002/14651858.CD004152.pub4. Review.

2. Antimicrobial resistance: global report on surveillance 2014 World Health Organisation (WHO), 2014. ISBN: 978924156474 8. http://www.who.int/ drugresistance/documents/surveillancereport/en/ (Accessed 10th June 2014)

Evidence-Based Dentistry (2014), 15, 58. doi: 10.1038/sj.ebd.6401030 